\title{
The Santoro III Massive Enterectomy: How Can We Justify the Risks in Obese Adolescents?
}

\author{
Thomas Inge • Michael Helmrath • Mark Vierra
}

Published online: 20 October 2010

(C) Springer Science+Business Media, LLC 2010

In this paper, Velhote describes short-term outcomes of ten adolescents who have undergone the Santoro III operation for weight loss. This operation involves a sleeve gastrectomy, a massive enterectomy (that may remove up to half of the small bowel length), an ileal Roux limb with gastroileostomy which creates a redundant gastric outlet and shunt to the ileum, and a jejunoileostomy leaving only $1.2 \mathrm{~m}$ in the absorptive stream. The operation is described as being neither restrictive nor malabsorptive, instead facilitating weight loss based on the "sound physiologic principles" of altering the neurohumoral response to feeding. It is intended to compensate for a modern diet to which our primitive gut and metabolism have not adapted. Outcomes have been previously reported in 228 adult patients from one center [1].

Velhote argues that "since childhood obesity begins early in life, the results of any weight loss operation must be maintained for a significantly longer time than in adults. Given this fact, perhaps more radical and definitive bariatric operations should be considered for adolescents, especially since medical surveillance could be minimized in the late postoperative period." For a number of reasons, we have very serious concerns about this logic and the performance of this procedure in adolescents.

First, the risks of this operation are considerable, and there is no reason to believe that long-term surgical risks or nutritional risks will be less with this procedure than with other more commonly performed weight loss operations. Short-term risks include all of the risks of a

\footnotetext{
T. Inge $(\bowtie) \cdot M$. Helmrath $\cdot M$. Vierra

Division of Pediatric General/Thoracic Surgery,

Cincinnati Children's Hospital Medical Center,

Cincinnati, OH, USA

e-mail: Thomas.Inge@cchmc.org
}

sleeve gastrectomy, as well as the risks of a laparotomy, small bowel resection, and gastroileal anastomosis. Longterm risks include all of the risks of a Roux-en-Y gastric bypass, including the long-term risk of anastomotic ulcer and the potentially catastrophic complication of an internal hernia - a real concern to patients of childbearing age [2], and particularly after half of the small bowel has been resected. No data are provided regarding long-term vitamin $B_{12}$ levels, iron absorption, calcium absorption, or bone metabolism (in adults or adolescents). We have trouble believing that it is safe to presume, as Velhote does, that long-term vitamin supplementation and meticulous metabolic surveillance are not necessary following massive small bowel resection and intestinal diversion. Adolescents may be at greater risk for symptomatic thiamine deficiency following weight loss surgery than adults [3]. We cannot conclude that this is a minor complication, or that this is a manifestation of preoperative thiamine deficiency as suggested by the authors. It would seem critical to understand in particular long-term fat-soluble vitamin metabolism, $\mathrm{B}_{12}$ and iron absorption, and bone metabolism before offering this operation to individuals who are still in the anabolic phase of somatic (particularly bone) development.

Second, it is not at all clear that the benefits of this operation are greater than the benefits of more commonly performed operations with which we have much greater experience and understanding. In fact, their reported weight loss is identical to that reported in adolescents undergoing gastric bypass surgery [4], and it may not be significantly different than the weight loss after sleeve gastrectomy [5] either.

We are particularly troubled by the assertion that this procedure might be preferable because it does not demand lifelong attention. Nothing could be further from the truth for any known weight loss operation. In particular, this 
would seem inadvisable for a complex gastrointestinal procedure that involves the intentional manipulation of gut hormones in young people without knowing the long-term sequela. Among the most serious complications of weight loss surgery is weight regain, an occurrence that ongoing surveillance is also intended to help prevent. Certainly until these issues are well understood in adults, it would seem inappropriate to apply these procedures to adolescents, for whom the ability to give informed consent for such an unusual and complicated procedure is questionable.

Finally, in this report, half of the adolescents who were subjected to Santoro III operations did not present with any identifiable comorbidity of obesity. We have strongly advocated for weight loss surgery as a treatment for adolescent morbid obesity to restore the health of the child [3]. For those adolescents who present with obesity and no coexisting medical conditions, an even less favorable riskbenefit ratio exists, compared to the adolescent who has an opportunity to enjoy reversal of a major comorbid health condition such as type 2 diabetes or obstructive sleep apnea.

While we can acknowledge that surgical innovation is critical to advances in health care [6], we must also realize that sometimes, more complex procedures that result in irreversible and massive sacrifice of bowel, with both predictable and unpredictable long-term adverse effects, cannot be blindly promoted as safe. Until we have a better understanding of these complex issues in adults we ought not to experiment on children. The use of a completely novel and complex procedure in adolescents without prior preclinical study, without controlled long-term adult outcome data, and without any clearly identifiable advantage over other modern operations is rationally unsound $[7,8]$.

Conflict of Interest The authors have no conflict of interest.

\section{References}

1. Santoro S, Milleo FQ, Malzoni CE, et al. Enterohormonal changes after digestive adaptation: five-year results of a surgical proposal to treat obesity and associated diseases. Obes Surg. 2008;18:17-26.

2. Gazzalle A, Braun D, Cavazzola LT, et al. Late intestinal obstruction due to an intestinal volvulus in a pregnant patient with a previous Roux-en-Y gastric bypass. Obes Surg. 2009 (in press)

3. Towbin A, Inge TH, Garcia VF, et al. Beriberi after gastric bypass surgery in adolescence. J Pediatr. 2004;145:263-7.

4. Inge TH, Jenkins TM, Zeller M, et al. Baseline BMI is a strong predictor of nadir BMI after adolescent gastric bypass. J Pediatr. 2010;156:103-8. e1.

5. Karamanakos SN, Vagenas K, Kalfarentzos F, et al. Appetite suppression and changes in fasting and postprandial ghrelin and peptide-YY levels after Roux-en-Y gastric bypass and sleeve gastrectomy: a prospective, double blind study. Ann Surg. 2008;247:401-7.

6. McCulloch P, Altman DG, Campbell WB, et al. No surgical innovation without evaluation: the IDEAL recommendations. Lancet. 2009;374:1105-12.

7. Caniano DA. Ethical issues in pediatric bariatric surgery. Semin Pediatr Surg. 2009;18:186-92.

8. Barkun JS, Aronson JK, Feldman LS, et al. Evaluation and stages of surgical innovations. Lancet. 2009;374:1089-96. 